\title{
Active Vertical-Coupler-Based Optical Crosspoint Switch Matrix for Optical Packet-Switching Applications
}

\author{
Riccardo Varrazza, Ivan B. Djordjevic, and Siyuan Yu
}

\begin{abstract}
Packet-switching characteristics are optimized across an integrated $4 \times 4$ optical crosspoint switch matrix consisting of active vertical-coupler-based switch cells. Optical gain difference between the shortest and the longest paths less than $3 \mathrm{~dB}$ is demonstrated. Bit error rate (BER) and power penalty measurements during packet routing have also been carried out over the entire 4 $\times 4$ matrix. At a 10-Gb/s packet data rate, a less than 1-dB power penalty is observed across the switch matrix, and the possibility for error-free packet routing is demonstrated with no BER floor observed.
\end{abstract}

Index Terms-Bit error rate (BER) performance, cross connect, integrated optoelectronics, optical crosspoint switch (OXS), optical packet switching, optical switch matrix, optoelectronics devices.

\section{INTRODUCTION}

$\mathbf{F}$ UTURE optical packet switching networks will require several key device technologies. One important technology is an optical crosspoint switch (OXS) fabric able to route optical packet [1]-[6] with sufficient switching speed. It has been theoretically shown that packet-switching networks will require an OXS reconfiguration time in the order of nanoseconds [7]. Good performances such as high extinction ratio, low polarization dependent loss, low loss, and good port count scalability are highly desirable.

It is generally agreed that electrooptic (EO) switching could be the mainstay of switching technologies for packet-based optical networks [4]. Compared with all-optical switching, it is technically more realistic while providing the necessary switching speed that microelectromechanical systems (MEMS) and thermal switches cannot deliver. It also provides the benefit of easier interfacing to network management functions, which is usually performed by a management layer in the electronics domain. Suitable materials for making optical switches include $\mathrm{LiNbO}_{3}$ for its field-induced EO effect and III-V semiconductors for their large and almost linear change of refractive index $(n)$ caused by the free-carrier plasma effect when carriers are injected in a particular area of the device [10] and their ability to turn a high-absorption coefficient into optical gain $(g)$ above the transparency carrier concentration $N_{t}$ in the proximity of the (direct) bandgap by the injection of the carriers. The possibility

Manuscript received December 18, 2003; revised April 16, 2004. This work was supported by the U.K. Engineering and Physical Sciences Research Council (EPSRC).

The authors are with the Department of Electrical and Electronic Engineering, University of Bristol, Bristol BS8 1TR, U.K. (e-mail: R.Varrazza@ bristol.ac.uk).

Digital Object Identifier 10.1109/JLT.2004.833259 of integration with other active devices such as semiconductor optical amplifiers (SOAs), detectors, and wavelength converters makes semiconductors even more attractive.

Many EO switch fabrics fall into the one of a few topologies based on $2 \times 2$ switch cells. For fast switching, the $2 \times$ 2 switches are often interferometer or directional coupler structures made in transparent substrate material or III-V semiconductors. EO effects (field induced or current/charge induced) are used to change the refractive index or phase shift of one of the arms in a waveguide interferometer or waveguide coupler to achieve switching. In general, this kind of switches has the advantage of low insertion loss not only because of the transparent nature of the material used, but also because that, in principle, the entire input signal is switched to the output without splitting if scattering and material background absorption losses are negligible. This makes them scalable in term of port count without introducing losses that scale with port count. The main drawback of an interferometer-based switching device (directional couplers can also be viewed as interferometers between supermodes) is the difficulty to achieve high extinction ratio and low crosstalk levels. This arises from the fact that in order to realize extremely low output levels at the unintended output port, the two interfering fields not only have to be exactly out of phase, but they also have to have exactly the same amplitude, with a misbalance of less than the required residual output levels (e.g., $-40 \mathrm{~dB}$ ). This puts a stringent requirement on coupler symmetry and therefore fabrication techniques. Typical values of crosstalk reported are in the order of -25 to $-30 \mathrm{~dB}$ [4]. This crosstalk level may not be low enough when port count is increased, particularly when many switching nodes are cascaded.

Another variety of popular optical switch fabrics, on the other hand, uses SOAs as optical ON-OFF switches or optical gates in a broadcast-and-select fabric. This relies on the modulation of optical absorption/gain of semiconductor materials. Because SOAs are highly absorptive to light when they are not injected with current, crosstalk levels can be significantly reduced and extinction ratio improved. Many examples have been reported [16]-[18] typically achieving an ON-OFF extinction ratio of $>40 \mathrm{~dB}$.

A major problem for SOA-gate-based switching fabric may be port-count scalability. This is reflected in two aspects. First, there is a fundamental split loss as a signal enters the fabric where it is divided into as many equal parts as the number of output ports, and only one part is routed to the desired output with the others lost. Clearly, every time port count doubles, this 
loss increases by $3 \mathrm{~dB}$. This directly results in rapid OSNR deterioration as amplified spontaneous emission (ASE) noise from the SOA is added to the signal. This problem can be somewhat alleviated by a staged fabric with input booster SOAs to compensate for the split loss. However, this leads to an increased number of expensive SOAs, as demonstrated in [17], and with them high power consumption due to the high number of SOAs simultaneously in the oN state.

It is therefore challenging to produce an optical switching mechanism that satisfies all the major requirements of speed, crosstalk, and scalability for optical packet-switching applications. The resulting switch device should also be able to provide low insertion loss, low path-dependent loss once connected into a switching fabric, and low polarization-dependent loss. Large-scale monolithic integration should also be possible. From this analysis, the advantages of refractive index $(n)$ modulation switches, mainly scalability, combined with the low-crosstalk ability of gain/absorption $(g)$ modulated switches, would produce a successful solution. This indicates a mechanism that adopts combined $g$ and $n$ modulation fabricated on a III-V semiconductor substrate material.

This paper presents a fast optical switch design based on active vertical couplers (AVCs) that puts above idea into practice. Section II describes the principle of operation, and the results achieved with individual switch unit that demonstrate the potential advantages of this technology. Section III describes the design of the multilayer semiconductor wafer structure in order to optimize the performance of a monolithic $4 \times 4$ switch matrix. The fabrication process is given in Section IV and followed in Section V by the characterizations of the $4 \times 4$ matrix switch where switching characteristics, path dependent loss, and transmission wavelength response are presented. $10 \mathrm{~Gb} / \mathrm{s}$ packet switching performances are presented in Section VI with bit error rate (BER) and power penalty results across the entire $4 \times 4$ matrix. Finally, Section VII summarizes and comments on future work.

\section{Active Vertical Coupler Based Optical Switch Unit}

\section{A. Device Topology}

As the switch units need to be connected to form larger switch fabrics, the main signal paths need to be passive (throughout this paper, "passive" means essentially optically transparent and "active" means absorptive or amplifying) optical waveguides so that optical signal can pass through a switch unit when it is not in use (OFF-state). Furthermore, in a switch fabric optical signals may pass through an unused switch unit in two different cases, either en-route from an input port to a switch unit being used (ON-state) down the line, or after being switched by a ON-state switch unit, en-route to an output port. In an all-passive waveguide switch structure this is never a problem, but in a structure with active (absorptive/amplifying) switching mechanisms, this leads to a topology illustrated in Fig. 1, in which an active optical waveguide optically coupled to both passive waveguides is used to form a local mechanism for bridging the optical signal between them.

The advantages of such an arrangement are obvious. Firstly, it can effectively suppress signal leakage when it is in the "OFF

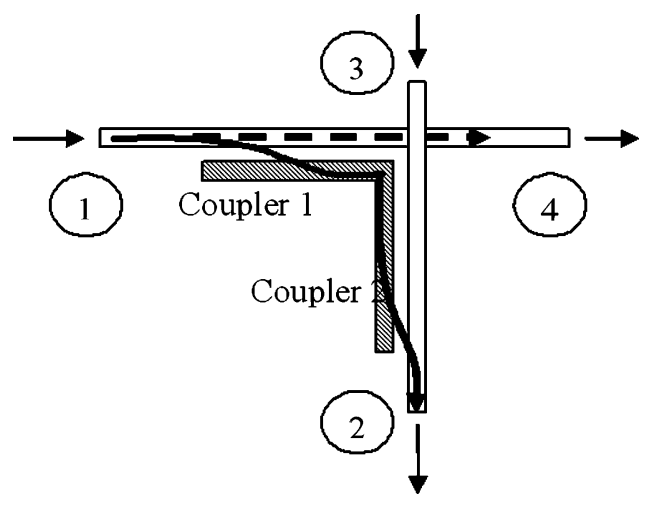

Fig. 1. Optical switch unit employing an active switching mechanism (shaded) and passive waveguides. Solid line: signal path in the ON-state, and dashed lines: signal paths in the OFF-state.

state. As commented earlier, when couplers 1 and 2 are disabled, the best achievable leakage levels at the end of a passive coupler is about $-30 \mathrm{~dB}$. However, because one arm of the waveguide coupler is now highly absorbing just as an SOA without current injection, extra attenuation of about $40 \mathrm{~dB}$ can be added to the leaked signal, thus possibly reducing leakage level to $-70 \mathrm{~dB}$. Second, it has maintained the integrity of input signal by transmitting essentially all its power to one output port without split loss, therefore is potential scalable. Third, optical gain attained in the active waveguide will allow compensation of losses and, more importantly, equalization of path dependent loss.

\section{B. Vertical-Coupler-Based Switch Unit and Matrix}

The device topology makes any coplanar photonic integration scheme quite difficult to fabricate, as passive and active waveguides has to be closely located (within about $1 \mu \mathrm{m}$ ) to form couplers. Switching structures based on the same principle was realized with regrowth techniques to achieve passive-active integration [20], using high precision ion implantation to confine injected carriers from leaking into adjacent waveguide areas.

On the other hand, this kind of structure is very suitable for realization in the vertical direction. Due to the high accuracy attainable by modern epitaxial techniques, forming a waveguide optical coupler in the vertical direction is realistic. Particularly, to fabricate uninterrupted passive waveguide in one layer and active waveguides in another layer is straightforward. The two passive waveguides will have to intersect each other if made in the same layer. This does introduce an additional source of crosstalk. However, this crosstalk has been proven to be sufficiently low [21] if waveguides cross each other at right angle.

A schematic illustration of a switch fabric based on above concept is illustrated in Fig. 2. The fabric consists of a grid of passive waveguides (light shade). At each crosspoint, two AVCs are formed between the passive waveguide and an upper layer of active waveguide (dark shade). A total internal reflection (TIR) [22] mirror vertically penetrates the active waveguide layer with an angle of $45^{\circ}$ with respect to the two couplers directions. This deflects the optical signal for $90^{\circ}$ from the input AVC to the output AVC. The switch cells conveniently connect with each other forming a matrix.

The injection of carriers into the active layer couplers turns the device into "ON state." It generates an optical gain in the 


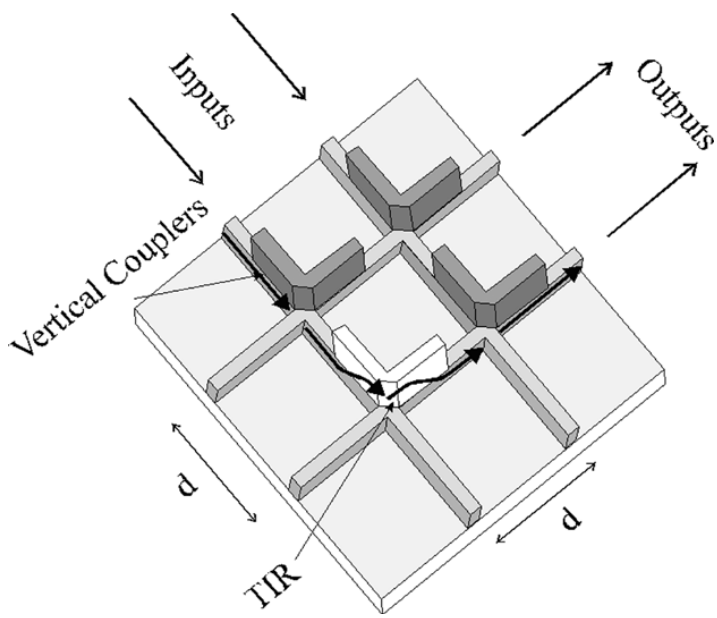

Fig. 2. Schematic illustration of AVC-based optical switch matrix.

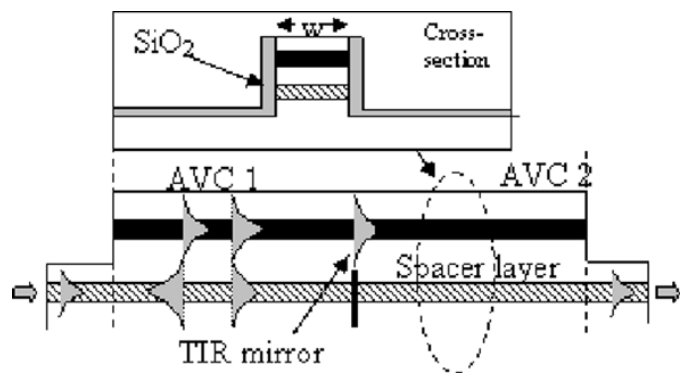

Fig. 3. Schematic illustration of the ON-state device model with the $\mathrm{I} / \mathrm{O}$ waveguide modes, the supermodes of the coupler, and the mirror aperture. A cross section is also given. The solid box indicates the active waveguide and the hashed box indicates the passive waveguide.

active waveguide and reduces its effective refractive index $n_{\mathrm{eff}}$ to a value matching that of the passive waveguide. In this situation, the input signal, launched into the input lower passive waveguide, is coupled up to the active upper waveguide and amplified in the first AVC and steered by the TIR mirror. Then, it is amplified again in the second AVC and coupled down to the passive waveguide and on to the cross output.

\section{DESIGN OF THE $4 \times 4$ OXS MATRIX}

As described previously and shown in Fig. 2, a $4 \times 4$ matrix is composed of 16 single switch cells interconnected by passive waveguides that perpendicularly intersect each other. At each crosspoint, each single switch cell is made of two AVCs. It is necessary to optimize the device parameters so that good performance of an entire switch matrix could be achieved. Most important, the path-dependent loss should be reduced. This consists of first optimizing single-unit transmission and subsequent equalization of transmission across the $4 \times 4$ matrix. In the work described in this paper, only transverse-electric (TE) transmission is considered.

\section{A. Optimization of Single Switch Unit}

Earlier devices [23]-[26] had been designed using a scalar passive waveguide model based on a code (FWAVE) developed in University Glasgow, Glasgow, U.K. Although they have demonstrated the principle of operation and a sufficiently

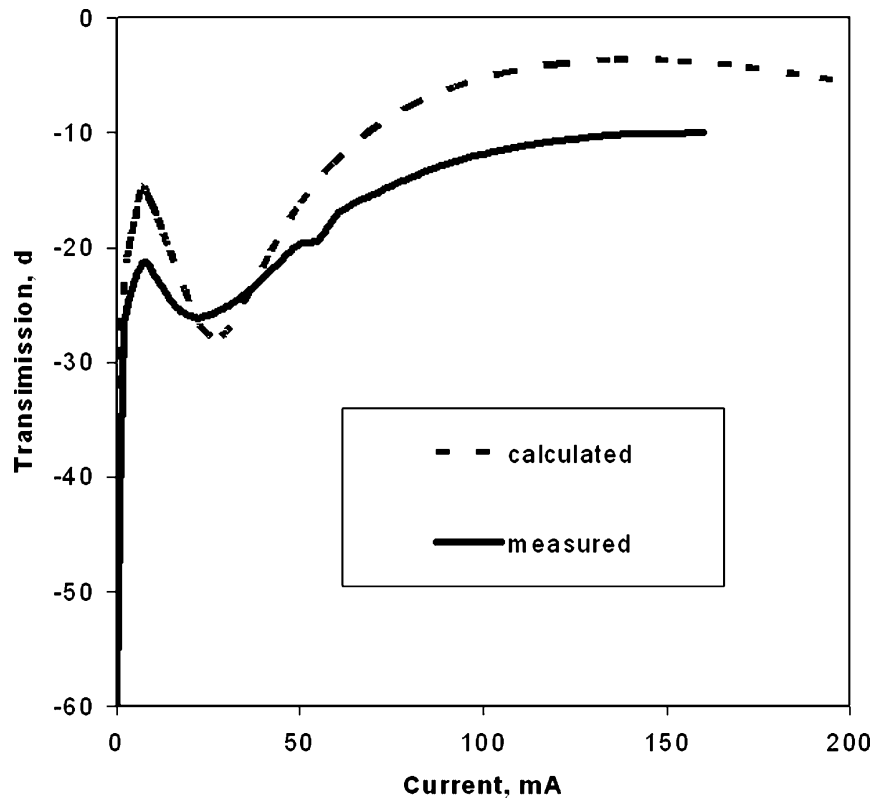

Fig. 4. Modeled and measured switching characteristics at $1550 \mathrm{~nm}$ for an AVC device with a 5-QW active layer, with waveguide width of $3 \mu \mathrm{m}$. The parameters used for the model are $d g / d N=3 \times 10^{-16} \mathrm{~cm}^{2}$ and $d n / d N=$ $2 \times 10^{-20} \mathrm{~cm}^{3}$.

fast switching speed (switching time $\sim 1.5 \mathrm{~ns}$ ), they were not able to achieve sufficiently low optical loss or optical gain. For optimized device design and simulation, a model using a vectorial waveguide solver (FIMMWAVE developed by Photon Design, Ltd.) to calculate the modes in waveguide coupler with complex refractive index profiles has been established.

The improved device model, as illustrated in Fig. 3, is based on deep-etched ridge waveguides of the width $(W)$ of 3 or $5 \mu \mathrm{m}$. The etch depth is such that it penetrates both waveguide layers, and the sidewall is passivated by an $\mathrm{SiO}_{2}$ layer. The device model consists of passive input/output (I/O) waveguide sections where the active layer and half the spacer layer are removed. The fundamental mode from the input waveguide excites the supermodes in AVC 1. The TIR mirror is simulated by an aperture that cuts off the field below the midpoint of the spacer layer at the end of the first AVC. This truncated field then excites the supermodes in AVC 2, which, in turn, with the field distribution at its end cross section, excites the output passive waveguide mode.

The computer model is validated against switching characteristics measured from previously fabricated devices. Fig. 4 shows a comparison between typical measured switching characteristics from previously reported [23] 5QW (quantum well) active layer devices and a modeled switching curve of the same structure. The agreement is good except for the absolute transmission values, where the measured values are approximately $5 \mathrm{~dB}$ lower than the calculated curve. This kind of discrepancy can be reasonably attributed to material losses that are not accounted for in the model and to fabrication related imperfections. It is important to note that the maxima and minima in both curves are arrived at almost the same current values. This is a good indication that the model reflects the coupling process in the AVC correctly, as these represent the maxima and minima of field amplitude moving past the end of the couplers as the refractive index is changed. The modeled curve dips below the measured characteristics at the minimum around $40 \mathrm{~mA}$. This could be 
because the actual depth of the TIR mirror is slightly deeper than midpoint of the spacer layer, therefore, reflecting more of the "tail" of the passive waveguide mode into the output AVC. This calculation also indicates that previous designs were not to achieve lossless switching due to nonoptimized design.

In design of new devices, we first optimize various multiplequantum-well (MQW) separate confinement heterojunction $(\mathrm{SCH})$ active waveguide structures so that maximum effective refractive-index modulation $\delta n_{\text {eff }}$ can be achieved for a different number of quantum wells. This is equivalent to maximization of modal gain in the same structures. Key material parameters to achieving correct design include the background refractive index $n_{0}$ of quaternary alloy semiconductor $\operatorname{In}_{x} \mathrm{Ga}_{1-x} \mathrm{As}_{y} \mathrm{P}_{1-y}$, the differential gain $d g / d N$, and differential refractive-index change $d n / d N$ in the QWs, as well as the monomolecular (A), radiative (B), and Auger (C) recombination coefficients in the QWs. Values of $\mathrm{A}=5 \times 10^{7}$, $\mathrm{B}=1 \times 10^{-10} \mathrm{~cm}^{-3}$, and $\mathrm{C}=1 \times 10^{-28} \mathrm{~cm}^{-6}$ are used. Adachi's model [27] is used to calculate the background material refractive-index dispersion data $n_{0}(\lambda)$ for various quaternary materials used. Away from bandgap, this has been shown to give good agreement with measured results. Close to and above bandgap, we use a broadening factor of $25 \mathrm{meV}$ for the oscillator [28]. Typical reported differential gain values for unstrained QWs are between 2 to $6 \times 10^{-16} \mathrm{~cm}^{2}$ and typical $d n / d N$ values at bandgap wavelength are about 2 to $3 \times 10^{-20} \mathrm{~cm}^{3}$ [29]. The background absorption in the QW at the bandgap wavelength is assumed to be $\alpha_{0}=5000 \mathrm{~cm}^{-1}$, and the transparent carrier density is assumed to be $1.5 \times 10^{18} \mathrm{~cm}^{-3}$. The refractive index $n$ and absorption $\alpha$ in the QWs are changed according to the formula

$$
\begin{aligned}
n(\lambda) & =n_{0}(\lambda)+\frac{d n}{d N} N \\
\alpha & =\alpha_{0}\left(1-\frac{N}{N_{t}}\right) \quad\left(\text { when } \quad N<N_{t}\right)
\end{aligned}
$$

and

$$
g=\frac{d g}{d N}\left(N-N_{t}\right) \quad\left(\text { when } \quad N>N_{t}\right)
$$

where $N$ is the carrier density in the QWs. $N$ at current density $J$ is decided by solving the steady-state carrier density rate equation

$$
\mathrm{A} N+\mathrm{B} N^{2}+\mathrm{C} N^{3}=\frac{\eta J}{e d_{q w} N_{q w}}
$$

where $e$ is electron charge, $\eta=0.8$ is the injection efficiency, $d_{\mathrm{qw}}$ is the thickness of each QW, and $N_{\mathrm{qw}}$ is the number of QWs. Carriers are assumed to be evenly distributed in all QWs.

The resulting $\delta n_{\text {eff }}$ and modal gain variation with injection current is shown in Fig. 5. It is clear that higher $N_{\mathrm{qw}}$ can produce larger $\delta n_{\text {eff }}$ at the ON-state current density limit of $10 \mathrm{kA} / \mathrm{cm}^{2}$, thereby allowing a more decoupled OFF-state coupler to be used. This will be beneficial to the reduction of residual coupling discussed in Section III-B. High $N_{\text {qw }}$ also provides more modal optical gain in the ON state, thereby enabling shorter devices to be made.

InGaAsP passive waveguide layer parameters are then decided according the achievable $\delta n_{\text {eff }}$ in the active waveguide

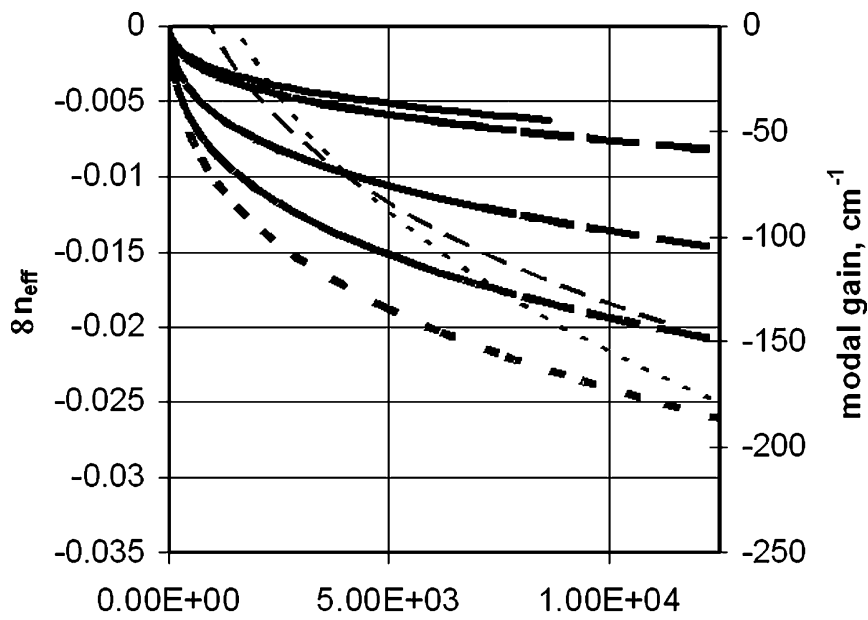

current density, $\mathrm{A} / \mathrm{cm}^{2}$

Fig. 5. Effective refractive-index change $\delta n_{\text {eff (thick lines) and modal gain }}$ of various MQW active layers as injection current density is increased. $\delta n_{\text {eff }}$ from top: 7QW with $d n / d N=1 \times 10^{-20} \mathrm{~cm}^{3} ; 10 \mathrm{QW}$ with $d n / d N=1 \times$ $10^{-20} \mathrm{~cm}^{3} ; 10 \mathrm{QW}$ with $d n / d N=2 \times 10^{-20} \mathrm{~cm}^{3} ; 10 \mathrm{QW}$ with $d n / d N=$ $3 \times 10^{-20} \mathrm{~cm}^{3}$; and 16QW with $d n / d N=3 \times 10^{-20} \mathrm{~cm}^{3}$. Modal gain: solid line $10 \mathrm{QW}$ with $d g / d N=3 \times 10^{-16} \mathrm{~cm}^{2}$; broken line: $16 \mathrm{QW}$ with $d g / d N=3 \times 10^{-16} \mathrm{~cm}^{2}$.

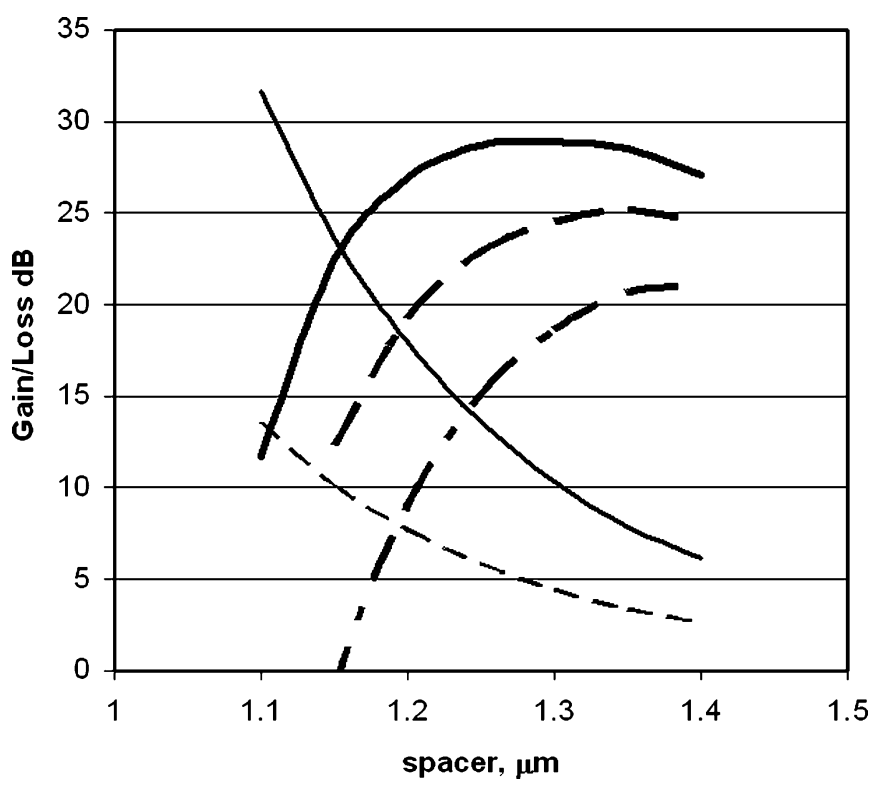

Fig. 6. Variation of ON-state transmission (thick lines) and OFF-state loss of a 7QW active layer $M \times M$ switch matrix (thin lines) in different switch paths. ON-state transmission from top: single switch cell (solid line); worst case on 4 $\times 4$ matrix (dashed line); and worst case on $8 \times 8$ matrix (dashed-dotted line). OFF-state loss: worst case on $4 \times 4$ matrix through six OFF-state cells (solid line), and worst case on $8 \times 8$ matrix through 14 OFF-state cells (dashed line).

layer so that equal $n_{\mathrm{eff}}$ is reached at the injection current limit. In order to reduce the material absorption loss associated with the band-edge absorption tail, the passive waveguide layer uses a bulk InGaAsP material with a bandgap wavelength of $\lambda_{g}=$ $1.20 \mu \mathrm{m}$.

For fixed coupler lengths and maximum ON-state current density, single switch units employing several different MQW active layers are optimized to give maximum ON-state transmission gain. This is achieved by adjusting the InP spacer layer thickness so that maximum signal optical power is obtained at the output of the device. An example is shown in Fig. 6, which 
plots the transmission of a 7QW switch unit (with coupler length of $450 \mu \mathrm{m}$ ) against the spacer layer thickness. Maximum transmission should be reached at a spacer thickness of $1.3 \mu \mathrm{m}$.

\section{B. Path-Dependent Loss and Transmission Equalization}

The key to achieving minimized path-dependent loss is reducing the transmission loss of signal through OFF-state switch units, i.e., loss between ports 1 and 4 or ports 3 and 2 in Fig. 1.

When in OFF state, signal transmission takes place in the passive waveguide below the active waveguide in one of the two AVCs. Apart from common reasons such as material, waveguide, and sidewall scattering losses, the AVC transmission loss is mainly caused by residual coupling of light into the active layer, which is now in a high absorption state.

Residual coupling exists despite that the $n_{\text {eff }}$ values of both waveguide layers are different. Directional coupler theory [29] indicates that this residual coupling can be reduced by two means, i.e., increasing the thickness of the spacer layer to reduce mode overlapping and increasing the difference between the $n_{\mathrm{eff}}$ values of the two waveguide layer. However, first the coupling length will increase exponentially with the spacer thickness [11]. Since the entire length of the AVC has to be pumped electrically to a certain carrier density to achieve the $\delta n_{\text {eff }}$ value for switching, the total injection current or power consumption increases with the coupler length. The target physical size of the integration chip also sets a limit to the length of couplers. Second, the available $\delta n_{\text {eff }}$ in the active layer is limited by the carrier density attainable in the MQW active layer. Therefore, too large a difference between $n_{\mathrm{eff}}$ values will theoretically require a very high current density to achieve, again resulting in high power consumption, and in practice being unrealistic due to thermal problems. A value of $10 \mathrm{kA} / \mathrm{cm}^{2}$ is chosen as the upper limit of operating current density. The target device sizes are chosen as $250 \times 250 \mu \mathrm{m}$ or $500 \times 500 \mu \mathrm{m}$ per switch unit to match standard fiber ribbon pitch. This limits the coupler length to about 200 and $450 \mu \mathrm{m}$, respectively.

Therefore, tradeoffs between the OFF-state loss, the AVC length, the injection current density, and power consumption has to be carefully considered.

The variation of both the single switch unit ON-state transmission $T_{\text {on }}$ and its OFF-state loss $L_{\text {off }}$ resulting from residual coupling is plotted in Fig. 6 for a fixed-length 7QW AVC device as the spacer layer thickness is changed. It is clear that the OFF-state loss reduces rapidly as the spacer thickness is increased, while the ON-state transmission peaks at a spacer thickness value of $1.3 \mu \mathrm{m}$ and then drops off. This peak corresponds to the optimum structure for a single switch unit or the shortest path in the switch matrix.

For an $M \times M$ matrix, the signal has to pass through a total of $2(M-1)$ OFF-state switch units if transmitted via the longest path. Therefore, it is important to maximize the ON-state transmission of this worst-case path

$$
T_{\min }=T_{\text {on }}-2(M-1) L_{\text {off }}
$$

which is also plotted in Fig. 6 for $M=4$ and $M=8$. It is clear that by increasing the spacer thickness beyond the $T_{\text {on }}$ maximum, the rapid reduction of $L_{\text {off }}$ results in a much smaller difference between $T_{\min }$ and $T_{\mathrm{on}}$ and therefore results in a $4 \times$ 4 matrix with reduced path-dependent gain. Further reduction of $L_{\text {off }}$ results in too much reduction of $T_{\text {on }}$, which may not be desirable from the noise-figure point of view. Therefore, a target OFF-state loss $L_{\mathrm{Off}}=0.5 \mathrm{~dB}$ is set for $M=4$. This will still result in 3-dB difference between worst- and best-path transmission in a $4 \times 4$ matrix. For the $M=8(8 \times 8)$ matrix, $L_{\text {off }}$ should be further reduced. Equalization within the $3-\mathrm{dB}$ path-dependent loss can simply be carried out by trimming the ON-state injection current to each switch unit in the matrix.

\section{FABRICATION PROCESS}

Several designs with two different cell sizes $\left(250 \times 250 \mu \mathrm{m}^{2}\right.$ and $500 \times 500 \mu \mathrm{m}^{2}$ ) have been generated. All the wafers are metal organic vapor phase epitaxy (MOVPE) grown. One of the resulting wafer structures has an intrinsic $\mathrm{SCH}$ active waveguide with $7 \times 75 \AA$ unstrained InGaAs QWs $\left(\lambda_{\mathrm{PL}}=1560 \mathrm{~nm}\right)$ separated by $60 \AA \mathrm{Q} 1.3$ barriers. The Q1.2 passive waveguide layer is $0.7 \mu \mathrm{m}$ thick, and the spacing layer thickness is $1.35 \mu \mathrm{m}$. This is processed into $4 \times 4$ switch matrices with $500 \times 500 \mu \mathrm{m}^{2}$ switch unit size and $450-\mu \mathrm{m}$ AVC length with 5- $\mu \mathrm{m}$ waveguide width. The 5- $\mu \mathrm{m}$ waveguide width is chosen for two reasons: 1) it is easier to achieve better relative mirror alignment accuracy (see the next paragraph), and 2) wider waveguides have higher active volume to sidewall surface area ratio, which reduces a proportion of surface recombination contribution to the total injection current. Narrower waveguide width can reduce switching current if these problems can be solved effectively.

The first critical fabrication issue is to achieve a straight smooth TIR mirror accurately aligned diagonally across the waveguide intersections by means of photolithography. A self-aligned two-level metal/dielectric masking scheme is used, in which the mirror and AVC etch mask is defined first in $\mathrm{SiO}_{2}$ by $\mathrm{CHF}_{3}$ reactive ion etching (RIE). A metal mask defining the waveguides is then carefully aligned on top of this dielectric mask layer, making sure that the TIR mirror cut across the waveguide cross within $\pm 0.25 \mu \mathrm{m}$. This alignment is made easier by the fact that 1 ) the $\mathrm{SiO}_{2}$ layer is sufficiently thin and 2 ) the critical alignment is reduced to one dimension along the TIR direction at a $45^{\circ}$ angle relative to the waveguides. The metal mask is then used to etch the $\mathrm{SiO}_{2}$ again so that the AVC waveguides and the passive waveguides are self-aligned.

The device structure in InP is then dry-etched in two steps using inductive-coupled plasma (ICP)/RIE in $\mathrm{Cl}_{2} / \mathrm{N}_{2}$ ambient [30]. The first etch depth equals to the finish ridge height of the passive waveguide sections. The metal mask is then removed by wet etching revealing the semiconductor surface in the passive waveguide sections and the $\mathrm{SiO}_{2}$ mask, which still covers the AVC waveguide. The sample is then etched in the second dry-etching step, which is critical as this defines the TIR mirror and the AVC structure. This etch step must therefore be extremely well controlled in sidewall verticality $\left( \pm 0.5^{\circ}\right)$, smoothness, bowing, notching, and depth (within $\pm 0.1 \mu \mathrm{m}$ of center of spacer thickness). Afterwards, the device is deposited with $200 \mathrm{~nm}$ of $\mathrm{SiO}_{2}$. Contact windows are opened on top of the AVCs using photolithography and $\mathrm{SiO}_{2}$ dry etching. Finally, a $\mathrm{Ti}-\mathrm{Pd}-\mathrm{Au}$ contact is sputtered on top of a patterned photoresist 


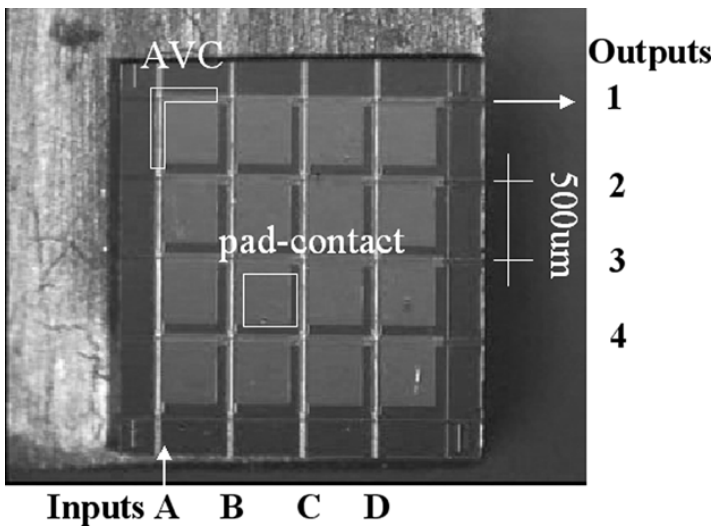

Fig. 7. Sample of the $4 \times 4$ matrix switch.

layer which is used to separate the p-contacts between switch units by liftoff. A finished $4 \times 4$ matrix is shown in Fig. 7 .

\section{The Matrix Switching Performance}

The $4 \times 4$ OXS matrix device used in this experiment is bonded p-side up to a copper heat sink (Fig. 7). A thermal electric cooler (TEC) controls the temperature of the heat sink at $20{ }^{\circ} \mathrm{C}$.

Extensive lasing was observed on as-cleave chips. After antireflective (AR) treatment of the unused back facets to $\sim 10^{-3}$ reflectivity, it has been observed that each switch cell has a lasing threshold that has a cavity that is path-length (input facet—switch cell—output facet) dependent, with the lowest threshold being observed with the shortest path of cell D1 (refer to Fig. 7), increasing gradually with increasing path length and forming a regular distribution across the device. After AR coating on the signal input/output (front) facets, the lasing threshold is increased to values higher than $550 \mathrm{~mA}$, confirming that lasing was due to Fabry-Pérot (FP) resonance between the input and output facets. Fig. 8 shows light output-current $(L-I)$ curves before and after front-facet AR treatment.

A setup shown in Fig. 9 is used to measure switching characteristics. A tunable laser source generates an input signal in the wavelength range of $1470-1580 \mathrm{~nm}$, which is modulated into a series of light pulses with a $1-\mu$ s pulsewidth synchronized to the variable amplitude switching current pulse. The light signal is coupled in and out of the $4 \times 4$ matrix using two conical fiber lenses. A polarization controller is used in order to produce TE-polarized input signal. At the output, the signal is filtered using a 3-nm optical bandpass filter to suppress the ASE noise. The fiber coupling loss in the setup is measured at about $9 \mathrm{~dB}$ per facet due to the highly elliptical waveguide near field, which can be improved by use of wedge lenses.

With the device in the OFF state (zero current), the measured on-chip leakage level was less than $-67 \mathrm{~dB}$ between all inputs and outputs, confirming the excellent crosstalk suppression in the switch matrix. Furthermore, no increase in leakage signal levels could be measured in all other outputs with any switch unit being switched into the ON state.

Each of the 16 switch units of a $4 \times 4$ matrix has been tested, and the average on-chip transmission is plotted in Fig. 10, with the error bars indicating the range of transmission. It is observed that all paths have achieved lossless switching or optical

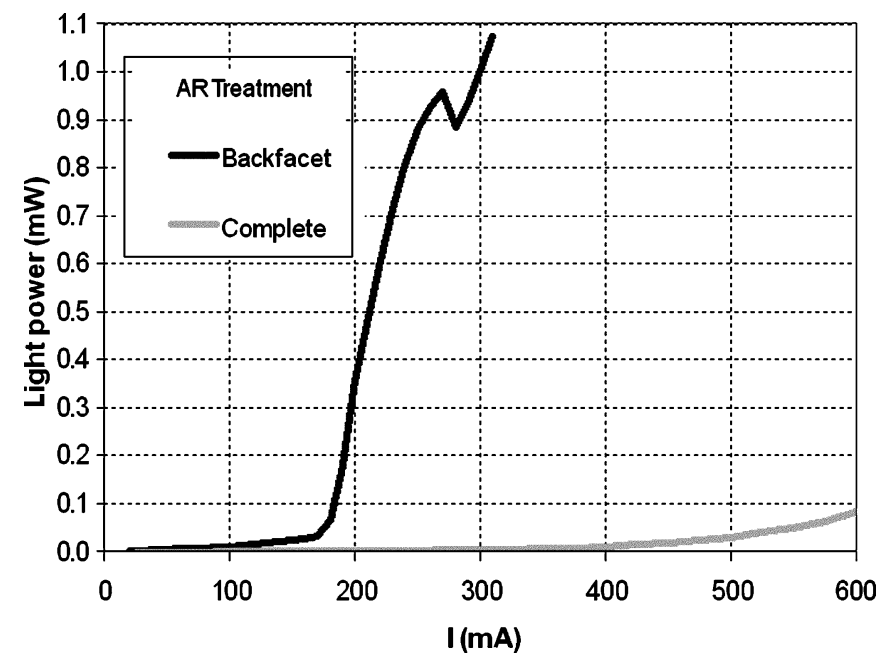

Fig. 8. $L-I$ curve measured before and after complete AR treatment.

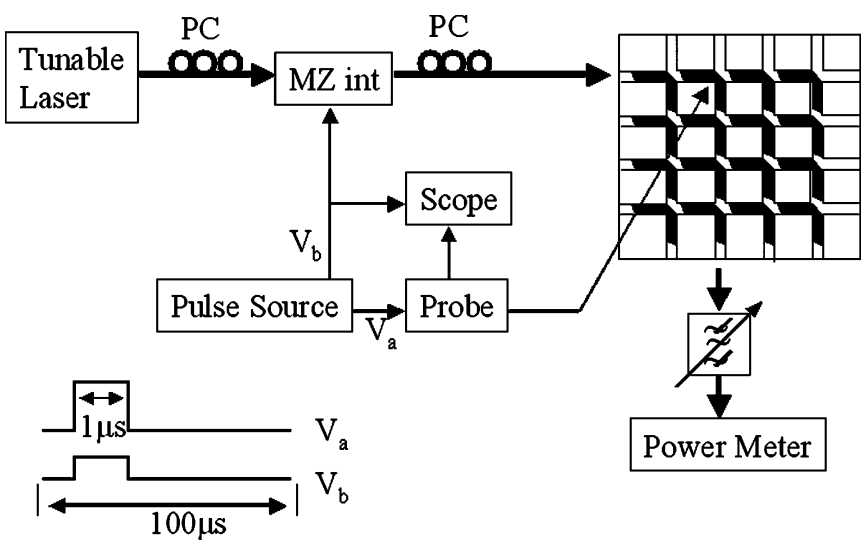

Fig. 9. Experimental setup for switching performance characterization.

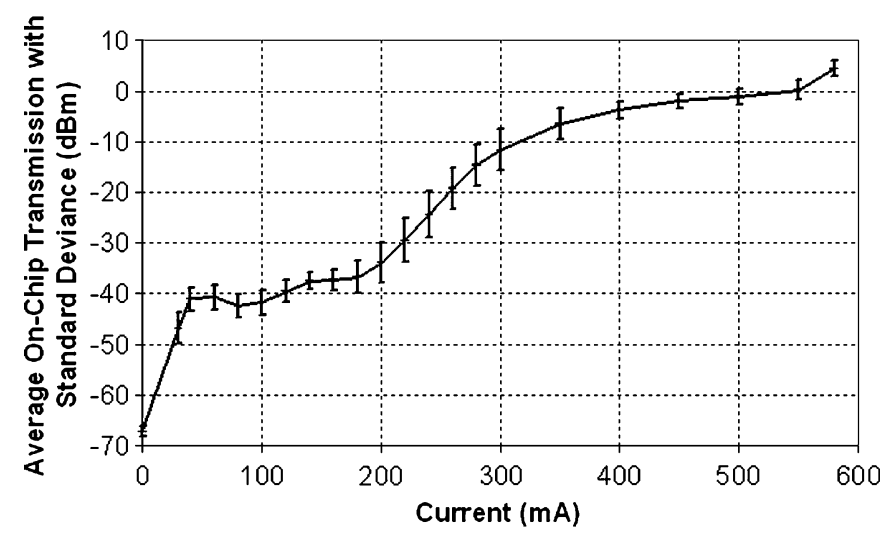

Fig. 10. A $4 \times 4$ OXS matrix average switching characteristic and standard deviation.

gain, and a transmission difference of less than $3 \mathrm{~dB}$ has been observed across the matrix at the ON-state current of $550 \mathrm{~mA}$ and above. This meets the design requirement of $0.5-\mathrm{dB} / \mathrm{cell}$ OFF-state loss. For most cells, currents higher than $550 \mathrm{~mA}$ result in some excessive FP resonance due to imperfect facet AR. However, in some units, higher gain was shown to be possible when current is further increased. An average ON-OFF contrast of near or above $70 \mathrm{~dB}$ is measured across the chip. 


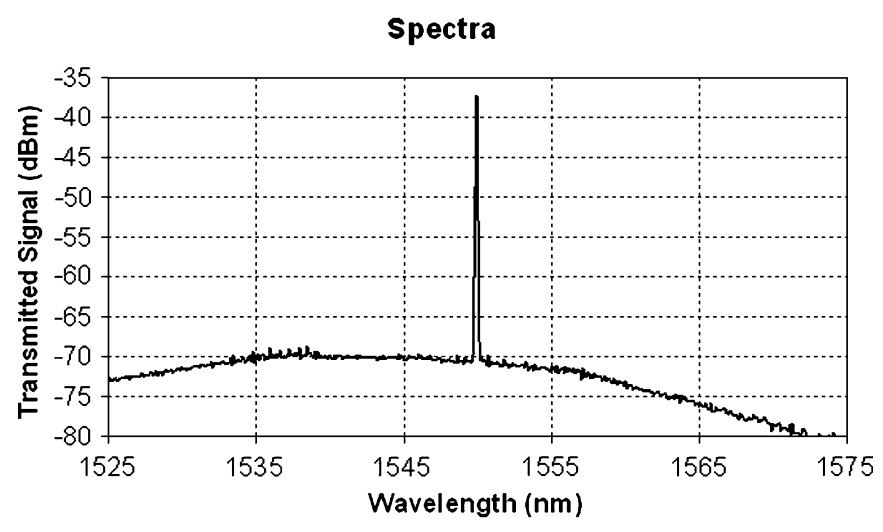

Fig. 11. Spectrum of $1550-\mathrm{nm}$ switched output signal, with $0-\mathrm{dBm}$ input power and $0.1-\mathrm{nm}$ resolution, measured at $1 \%$ duty circle.

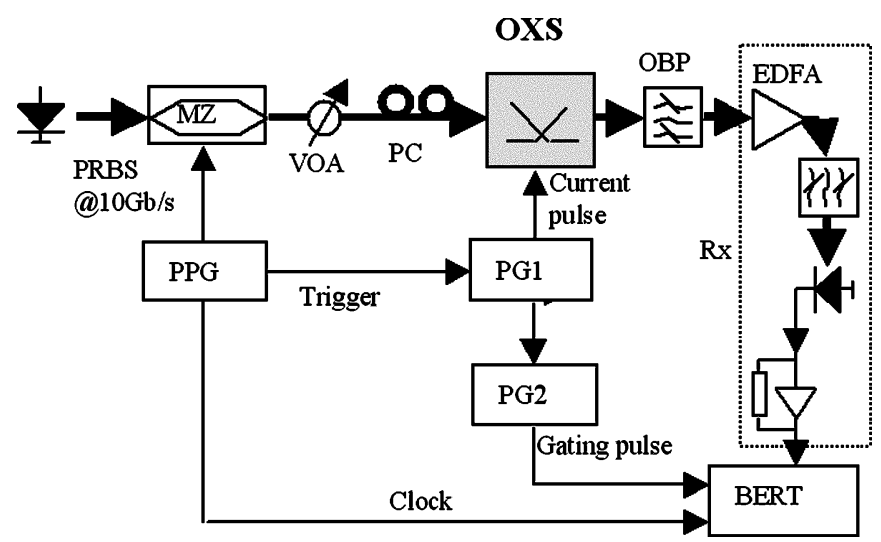

Fig. 12. BER measurement setup. PC: Polarization controller; MZ: MachZehnder modulator; VOA: variable optical attenuator; OXS: optical crosspoint switch; OBP: optical bandpass filter; Rx: receiver; PPG: pulse patlem generator; PG: pulse generator; BERT: BER detector.

At $0-\mathrm{dBm}$ input signal level, the unfiltered output spectrum is characterized by a switched signal peak of $34 \mathrm{~dB}$ above the device ASE floor (Fig. 11). A gain-wavelength response measurement observes a $3-\mathrm{dB}$ optical bandwidth of $20 \mathrm{~nm}$ in the $C$ band. This can be improved by better AR coating suppressing the residual FP ripples.

\section{Packet-Switching Mode BER PERFORMANCE AT $10 \mathrm{~Gb} / \mathrm{s}$}

Packet-switching performance has been assessed with BER measurements at a packet data rate of $10 \mathrm{~Gb} / \mathrm{s}$.

The experimental setup is shown in Fig. 12. A continuouswave laser source, operating at 1550-nm wavelength, is externally modulated using a Mach-Zehnder modulator by a continuous pseudorandom bit sequence (PRBS) of length $2^{15}-1$ at a bit rate of $10 \mathrm{~Gb} / \mathrm{s}$ from a pulse-pattern generator (PPG). The optical data passed through the polarization controller are coupled to the OXS by a fiber lens. The continuous optical data stream is chopped into packets by the switching operation of the switch cell being tested, which are driven by current pulses generated by PG1 and synchronized to a trigger pulses provided by the PPG at the start of every $2^{5}=32$ PRBS cycles. The data packets so formed are forwarded to the corresponding output port and again coupled into fiber by a second fiber lens. The optical bandpass filter that follows the OXS is used to suppress the ASE noise.
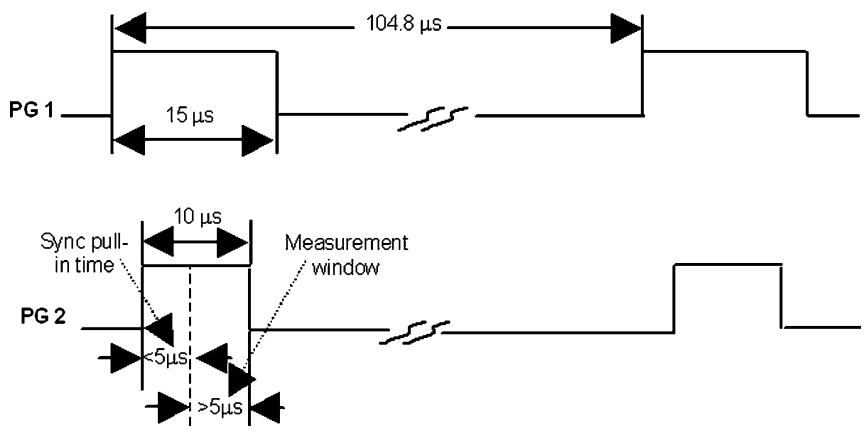

Fig. 13. Packet/burst timing diagram. PG1: Switching pulses; PG2: gating pulses.

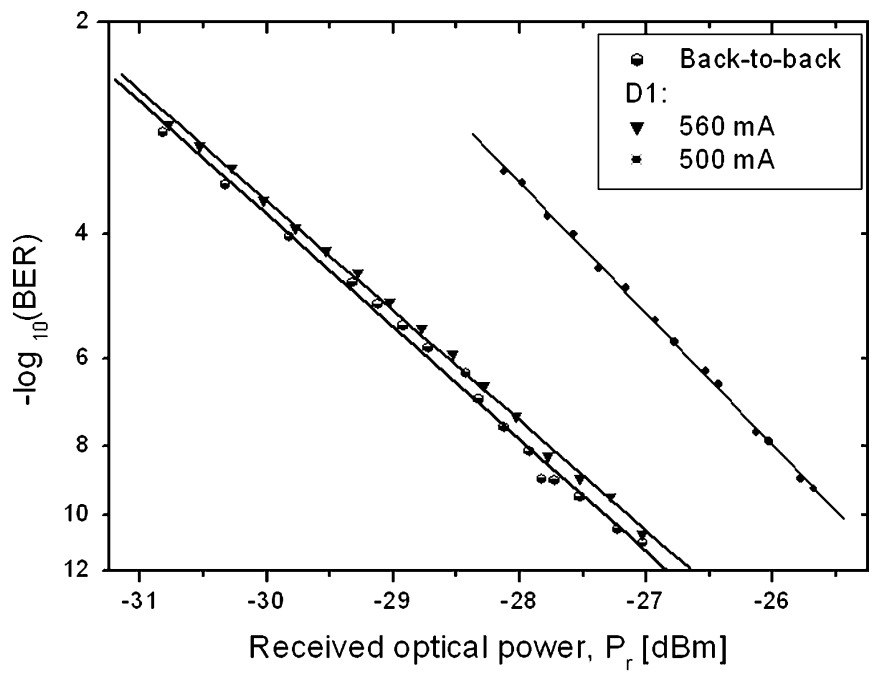

Fig. 14. Gated-mode BER performances of the shortest path or single switch unit.

The 10-Gb/s optically amplified receiver consists of an erbium-doped fiber amplifier (EDFA) used as a preamplifier and a 12-GHz bandwidth photoreceiver with its transimpedance amplifier output connected to the BER detector (BERT). The BER detector is operated in a gated mode, clocked by a gating pulse generated by PG2 that is slaved to PG1 and therefore synchronized to the data packet. The length of the BER test window is determined by this gating pulse, which is properly delayed relative to the switching pulse to avoid detecting the degraded signal during the switch-ON and -OFF edges (which in true packet-switching applications should usually coincide with guard times). The packet envelope and BER detector gating timing and packet interval are illustrated in Fig. 13. The duration of each packet is $15 \mu \mathrm{s}$, while the BER detector gating pulse is $10 \mu \mathrm{s}$ wide. Part of the gating pulse duration $(<5 \mu \mathrm{s})$ is used by the BERT to achieve synchronization. The remaining gives a BER measurement time window of $>5 \mu \mathrm{s}$, ensuring coverage for the complete PRBS cycle of $3.276 \mu \mathrm{s}$. Packets are switched every $104.8 \mu \mathrm{s}$.

The BER measurements are carried out on all 16 cells in the $4 \times 4$ OXS matrix by varying the input power to the OXS using the VOA.

The BER measurement results for the shortest path D1 (see Fig. 7) for two switching current amplitudes, as well as of the back-to-back configuration, are shown in Fig. 14. It can be seen that the power penalty is reduced with increasing switching current. At $560 \mathrm{~mA}$, the BER performance approaches that of the 


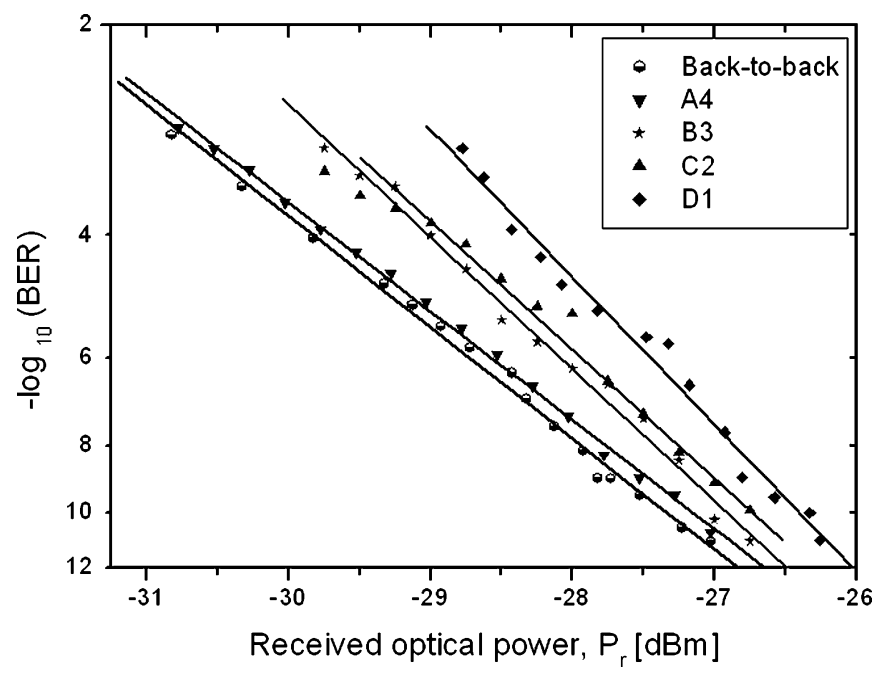

Fig. 15. Gated-mode BER performances of switching units on the diagonal of the matrix.

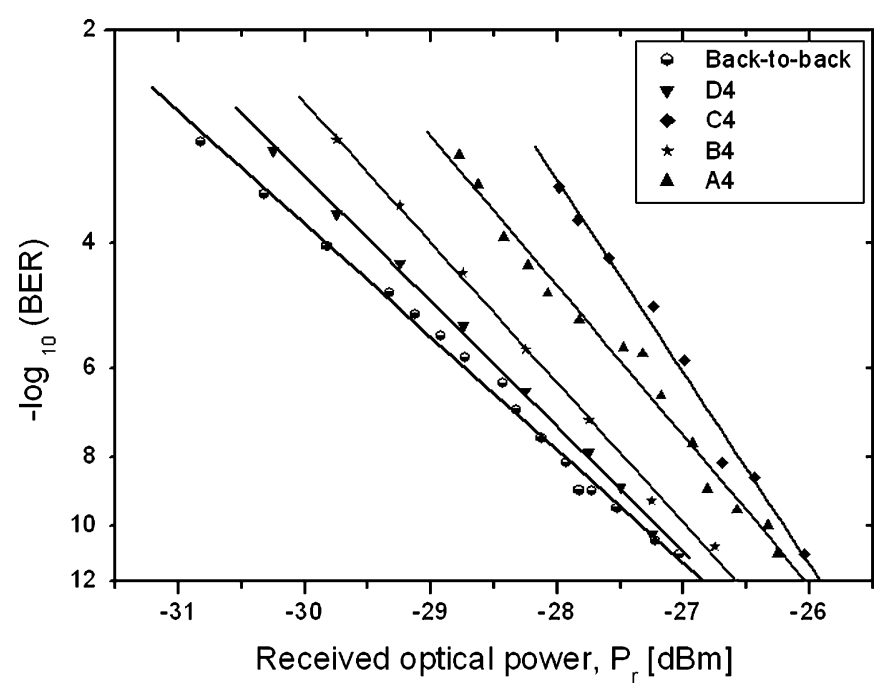

Fig. 16. Burst-mode BER performance of the left-most column switching unit.

back-to-back configuration with a penalty of about $0.2 \mathrm{~dB}$ at a BER of $10^{-9}$. Throughout the experiments, the accuracy of power measurement is estimated to be within $0.3 \mathrm{~dB}$. The current of the switch cells could not be increased further because of increased FP resonance (due to nonideal AR treatment of facets) and related gain spectral fluctuations, which results in patterning in the output packet data.

In Fig. 15, the gated BER performance of switching units along the diagonal of the matrix (paths D1, C2, B3, and A4) is shown. At BER $=10^{-11}$, power penalties of less than $1 \mathrm{~dB}$ are observed for all diagonal switch cells, with larger penalties for longer paths as expected due to the existence of residual path-dependent loss. The spread of the power penalty agrees with the path-dependent loss reasonably, considering that half the 3-dB path-dependent loss happens prior to the switching unit therefore impact on the OSNR is about $1.5 \mathrm{~dB}$. In Fig. 16, the burst BER performance for the switching units on the same array (paths A4, B4, C4, and D4) is shown. Similarly, at a BER of $10^{-11}$, power penalties less than $1 \mathrm{~dB}$ are again observed for the entire column. For all cells in the matrix, an error-free packetrouting operation has been achieved without any BER floor.

\section{CONCLUSION AND DISCUSSIONS}

This paper has presented the design rationale, optimization, fabrication, and characterization of a monolithic integrated $4 \times$ 4 optical switch matrix. By employing optical switching units based on InGaAsP-InP active vertical coupler structures, the switch matrix has demonstrated an ON-OFF extinction ratio as high as $70 \mathrm{~dB}$ and extremely low crosstalk levels of $<-65 \mathrm{~dB}$. Lossless switching with optical gain up to $5 \mathrm{~dB}$ is achieved across the matrix. BER and path-dependence transmission across the switch matrix has been minimized, which results in power penalty uniformity of within $1 \mathrm{~dB}$ across the matrix at $10-\mathrm{Gb} / \mathrm{s}$ optical-packet-mode operations. Error-free operation without BER floor is demonstrated for transmission through all switch paths across the entire matrix. These good performances, combined with previously demonstrated switching speeds in the order of $\sim 1.5 \mathrm{~ns}$, make this device a promising one for future high-speed, highly flexible all-optical packet-switching networks.

However, before application in practical networking environment, several key issues have to be further addressed. Most important, the polarization-dependent loss must be minimized. Polarization dependence in the AVC switch unit arises from two sources: the polarization dependence of the coupling and that of the optical gain. It has been demonstrated that vertical couplers can be made polarization independent [31], and polarizationindependent optical gain structure is well reported in SOAs. Improvements in device structure and fabrication quality should also be made in order to minimize additional optical loss caused by fabrication defects (such as TIR mirror and waveguide sidewall scattering loss due to striations) as well as to reduce the carrier loss through surface recombination in a deep-ridge structure. Heating due to high switching currents needs to be reduced by using narrower waveguides and effective thermal management.

\section{ACKNOWLEDGMENT}

The authors would like to thank the U.K. Engineering and Physical Sciences Research Council (EPSRC) for funding this work, the EPSRC III-V semiconductor facility in the University of Sheffield, Sheffield, U.K., for providing metal organic vapor phase epitaxy (MOVPE) wafer growth service, the Oxford Instrument Plasma Technologies for providing very helpful support for high-quality III-V semiconductor etching, the British Aerospace Innovation Laboratories for providing technical assistance in BER measurement, and Prof. I. H. White of the University of Cambridge, Cambridge, U.K., for his involvement in early works and for useful discussions.

\section{REFERENCES}

[1] E. Iannone and R. Sabella, "Optical path technologies: a comparison among different cross-connect architectures,' J. Lightwave Technol., vol. 14, pp. 2184-2196, Oct. 1996.

[2] M. Duelk, J. Gripp, J. Simsarin, A. Bhardwaj, P. Bernasconi, M. Zirngibl, and O. Laznicka, "Fast packet routing in a $2.5 \mathrm{~Tb} / \mathrm{s}$ optical switch fabric with $40 \mathrm{~Gb} / \mathrm{s}$ duobinary signal at $0.8 \mathrm{~b} / \mathrm{s} / \mathrm{Hz}$ spectral efficiency," in Proc. Optical Fiber Communication Conf. Exposition, 2003, Postdeadline paper, pp. PD8-1-PD8-3.

[3] D. J. Blumenthal, J. E. Bowers, L. Rau, C. Hsu-Feng, S. Rangarajan, W. Wang, and K. N. Poulsen, "Optical signal processing for optical packet switching networks," IEEE Commun. Mag., vol. 41, pp. S23-S29, Feb. 2003.

[4] G. I. Papadimitriou, C. Papazoglou, and A. S. Pomportsis, "Optical switching: Switch fabrics, techniques, and architectures," J. Lightwave Technol., vol. 21, pp. 384-405, Feb. 2003. 
[5] N. Chi, J. Zhang, and P. Jeppesen, "All-optical subcarrier labeling based on the carrier suppression of the payload," IEEE Photon. Technol. Lett., vol. 15, pp. 781-783, May 2003.

[6] S. J. B. Yoo, H. J. Lee, Z. Pan, J. Cao, Y. Zhang, K. Okamoto, and S. Kamei, "Rapidly switching all-optical packet routing system with optical-label swapping incorporating tunable wavelength conversion and a uniform-loss cyclic frequency AWGR," IEEE Photon. Technol. Lett., vol. 14, pp. 1211-1213, Aug. 2002.

[7] D. Sadot and I. Elhanany, "Optical switching speed requirements for terabit/second packet over WDM networks," IEEE Photon. Technol. Lett., vol. 12, pp. 440-442, Apr. 2000.

[8] H. Murata, M. Izutsu, and T. Sueta, "All-optical switching in novel waveguide X-junctions with localized nonlinearity," IEICE Trans. Electron., vol. E82C, no. 2, pp. 321-326, Feb. 1999.

[9] M. Renaud, M. Bachmann, and M. Erman, "Semiconductor optical space switches," IEEE J. Select. Topics Quantum Electron., vol. 2, pp. 277-288, June 1996.

[10] B. R. Bennet, R. A. Soref, and J. A. Del Alamo, "Carrier-induced change in refractive index of InP, GaAs, and InGaAsP," IEEE J. Quantum Electron., vol. QE-26, pp. 113-122, Jan. 1990.

[11] L. A. Coldren and S. W. Corzine, Diode Lasers and Photonics Integrated Circuits. New York: Wiley, 1995.

[12] S. Murata, A. Tomita, and A. Suzuki, "Influence of free carrier plasma effect on carrier-induced refractive index change for quantum-well lasers," IEEE Photon. Technol. Lett., vol. 5, pp. 16-19, Jan. 1993.

[13] G. P. Agrawal and N. K. Dutta, Semiconductor Lasers-Second Edition. New York: Van Nostrand, 1993.

[14] M. K. Pandit, H. P. Chan, K. S. Chiang, S. Ghosh, and A. K. Das, "A digital optical switch (DOS) in polymer using truncated-structural X-branches (TSXB)," Microwave Optical Technol. Lett., vol. 27, no. 4, pp. 229-233, Nov. 20, 2000.

[15] M. N. Khan, B. I. Miller, E. C. Burrows, and C. A. Burrus, "Highspeed digital Y-branch switch/modulator with integrated passive tapers for fiber pigtailing," Electron. Lett., vol. 35, no. 11, pp. 894-896, May 27, 1999

[16] T. Kato, J. Sasaki, T. Shimoda, H. Hatakeyama, T. Tamanuki, S. Kitamura, M. Yamaguchi, T. Sasaki, K. Komatsu, M. Kitamura, and M. Itoh, "Hybrid integrated $4 \times 4$ optical matrix switch module on silica based planar waveguide platform," IEICE Trans. Electron., vol. E82-C, no. 2, pp. 305-312, 1999.

[17] C. P. Larsen and M. Gustavsson, "Linear crosstalk in $4 \times 4$ semiconductor optical amplifier gate switch matrix," J. Lightwave Technol., vol. 15, pp. 1865-1870, Oct. 1997.

[18] B. C. Qiu, X. F. Liu, M. L. Ke, H. K. Lee, A. C. Bryce, J. S. Aitchison, J. H. March, and C. B. Button, "Monolithic fabrication of $2 \times 2$ crosspoint switches in InGaAs-InAlGaAs multiple quantum wells using quantumwell intermixing," IEEE Photon. Technol. Lett., vol. 13, pp. 1292-1294, Dec. 2001.

[19] T. Kirihara, M. Ogawa, H. Inoue, and K. Ishida, "Lossless and lowcrosstalk characteristics in an InP-based $2 \times 2$ optical switch," IEEE Photon. Technol. Lett., vol. 5, pp. 1059-1061, Sept. 1993.

[20] G. A. Fish, L. A. Coldren, and S. P. DenBaars, "Suppressed modal interference switches with integrated curved amplifiers for scaleable photonic crossconnects," IEEE Photon. Technol. Lett., vol. 10, pp. 230-232, Feb. 1998.

[21] M. Owen, "Monolithic integration of optical space switches," Ph.D. dissertation, Dept. of Electronics and Electrical Engineering, Bristol University, Bristol, U.K., 1998.

[22] L. Faustini, C. Coriasso, A. Stano, C. Cacciatore, and D. Campi, "Loss analysis and interference effect in semiconductor integrated waveguide turning mirrors," IEEE Photon. Technol. Lett., vol. 8, pp. 1355-1357, Oct. 1996.

[23] S. Yu, R. Varrazza, M. Owen, R. V. Penty, I. H. White, D. Rogers, S. Perrin, and C. C. Button, "Ultra-low crosstalk, compact integrated optical crosspoint space switch arrays employing active InGaAsP/InP vertical waveguide couplers," presented at the Conf. Lasers Electro-Optics (CLEO99), Baltimore, May 1999, Postdeadline paper PD24.

[24] S. Yu, M. Owen, R. Varrazza, D. Rogers, S. Perrin, C. C. Button, R. V. Penty, and I. H. White, "Compact integrated optical crosspoint switches employing active InGaAsP/InP vertical waveguide couplers with ultralow crosstalk," in Proc. Eur. Conf. Optical Communications (ECOC99), vol. I, Sept. 1999, pp. 126-127.

[25] S. Yu, M. Owen, R. Varazza, R. W. Penty, and I. H. White, "High speed optical packet routing demonstration of a vertical coupler crosspoint space switch array," in Proc. Conf. Lasers Electro-Optics (CLEO2000), 2000, pp. 256-257.
[26] R. Varrazza, A. Wonfor, S. Yu, B. Cakmak, T. V. Penty, and I. H. White, "Demonstration of simultaneous packet routing and wavelength conversion at $10 \mathrm{~Gb} / \mathrm{s}$ in a highly compact, lossless vertical coupler space switch," in Proc. Eur. Conf. Optical Communications (ECOC2000), vol. 4, Sept. 2000, p. 67

[27] S. Adachi, Physical Properties of III-V Semiconductor Compounds, InP, InAs, GaAs, GaP, InGaAs, and InGaAsP. New York: Wiley, 1992.

[28] C. Tanguy, "Refractive index of direct bandgap semiconductor near the absorption threshold: Influence of excitonic effects," IEEE J. Quantum Electron., vol. 32, pp. 1746-1751, Oct. 1996.

[29] D. Marcuse, Theory of Dielectric Optical Waveguides-2nd Ed. Boston, MA: Academic, 1991.

[30] R. Varrazza, L. Deng, A. Goodyear, and S. Yu, "H-free anisotropic InP ICP etching with controllable etch rate," presented at the Dry Etching-Advances Trends Conf., London, U.K., Jan. 22, 2003.

[31] R. J. Deri, F. G. Patterson, and S. P. Dijaili, "Birefringence compensation for polarization-independent directional coupler wavelength filters," IEEE Photon. Technol. Lett., vol. 7, pp. 376-378, Apr. 1995.

[32] T. Ito, N. Yoshimoto, K. Magari, and H. Sugiura, "Wide-band polarization-independent tensile-strained InGaAs MQW-SOA gate," IEEE Photon. Technol. Lett., vol. 10, pp. 657-659, May 1998.

Riccardo Varrazza, photograph and biography not available at the time of publication.

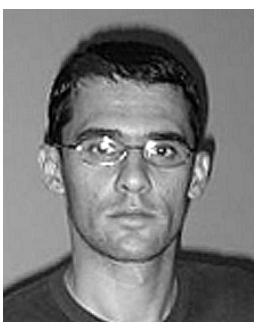

Ivan B. Djordjevic received the Dipl.Ing., M.S., and Ph.D. degrees, all in electrical engineering, from the University of Nish, Nish, Serbia, in 1994, 1997, and 1999, respectively.

From 1994 to 1996, he was with the Faculty of Electronic Engineering, University of Nish, working on modeling and simulation of optical/digital communication systems. From 1996 to 2000, he was with the State Telecommunications Company (Serbia Telecom), District Office for Networks, Nish, Serbia, where he was involved in digital transmission systems commissioning and acceptance, design, maintenance, installation, and connection. From 2000 to 2001, he was with the National Technical University of Athens, Greece, and with TyCom US, Inc. (now TyCo Telecommunications), USA, where he was involved in modeling and simulation of dense-wavelength-division-multiplexing (DWMD) systems. During 2002 and 2003, he was with the University of Arizona, Tucson; the University of Bristol, U.K.; and the University of West of England, Bristol, U.K., working on forward-error correction and iterative decoding for optical transmission, optical CDMA, high-speed transmission, and optical switches. $\mathrm{He}$ is now with the University of Arizona, on leave from the University of West of England. He is an author of more than 35 international journal papers and more than 45 international conference papers. His research interests include DWDM fiber-optic communication systems and networks, FEC for optical communications, optical CDMA, optical packet switching, coding theory, coherent optical communications, and statistical communication theory.

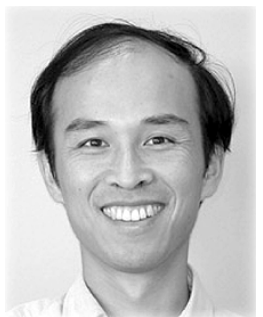

Siyuan Yu was born in Nanchang, Jiangxi Province, China, in May 1963. He received his B. Eng. degree from Tsinghua University, Beijing, China, in 1984, the M. Eng. degree from Wuhan Research Institute of Post and Telecommunications, Wuhan, China, in 1987, where he worked on the frequency stabilization of semiconductor lasers, and the Ph.D. degree in electronics and electrical engineering from the University of Glasgow, Glasgow, U.K., in 1997, where he studied monolithically integrated mode-locked semiconductor ring lasers.

He joined the Department of Optoelectronic Engineering, Huazhong University of Science and Technology in 1987 and worked on semiconductor optical amplifiers and other optoelectronic devices. In 1996, he joined the Department of Electrical and Electronic Engineering, University of Bristol, Bristol, U.K., where he is currently a Senior Lecturer. His current research interests are photonic devices in optical networks including optical packet switches, tunable lasers, wavelength converters, and all-optical switches. He is the author of more than 40 papers and inventor or co-inventor of one Chinese patent and four United Kingdom and international patents. 